\title{
Clear Air Turbulence: Problems and Solutions
} (A State-of-the-Art Report)

\author{
by \\ Elmar R. Reiter
}

\author{
Technical Paper No. 77 \\ Department of Atmospheric Science \\ Colorado State University \\ Fort Collins, Colorado
}

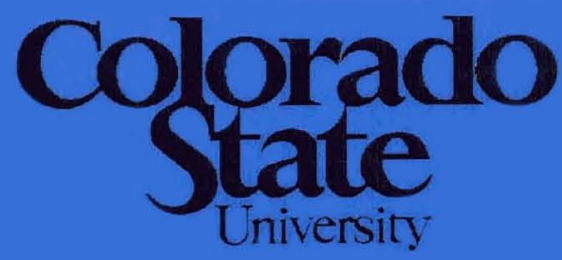

\section{Department of \\ Atmospheric Science}

\author{
Paper No. 77
}




\section{Clear Air Turbulence: Problems and Solutions (A State-of-the-Art Report)}

Elmar R. Reiter

Department of Atmospheric Science, Colorado State University

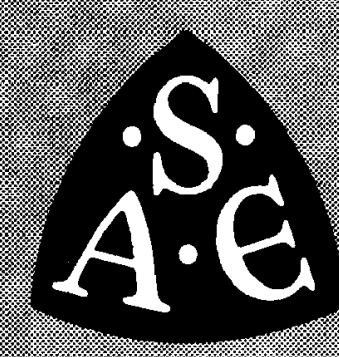


A summary is given of the present state of knowledge on the physical causes of clear-air turbulence (CAT). Special reference is made to recent measurement results from Project TOPCAT. Implications of these flight investigations, which were made over Australia, on remote sensing of $\mathrm{CAT}$ and on the planning of future research efforts are outlined.

\section{Clear Air Turbulence: Problems and Solutions (A State-of-the-Art Report)}

\author{
Elmar R. Reiter \\ Department of Atmospheric Science, Colorado State University
}

AFTER MORE THAN A DECADE of research on CAT, it still remains one of the much talkedabout hazards of high-altitude jet aviation. In spite of all the efforts that have gone into numerous studies, we cannot yet boast of any skill in its accurate prediction. Only very recently have we gained some knowledge on the mechanism of its formation.

CAT may be regarded as the result of atmospheric turbulence acting on an airborne vehicle (aircraft or missile). Since the presence of CAT is tied to the presence of a vehicle experiencing bumpiness, the response characteristics of this vehicle play an important role. This explains why the frequency of CAT encounter increased with the development of faster-flying jet aircraft. More appropriately, CAT should be called "bumpiness in flight through clear air". We will, however, adhere to the established nomenclature and not attempt to introduce a new tongue twister.

In considering CAT we normally rule out low -level convectively induced turbulence, turbulence in, or near, clouds, and turbulence within the friction layer near the ground.

\section{CHARACTERISTICS OF CAT}

There are no absolutely "safe" regions in the atmospheric layers presently used by aircraft in which no bumpiness may occur at all. Statistical investigations by Bannon (1)*, Clodman et al. (2), Colson $(3,4)$ and many

* Numbers in parentheses designate References at end of paper. others revealed that the more violent forms of CAT are associated with certain regions and with certain flow patterns aloft. Bannon's classical pattern of CAT distribution around the jet stream still remains essentially unchanged, indicating a relatively high frequency of occurrency on the cyclonic side of, above and below, the jet-stream core. In these regions the atmosphere is generally thermally stable, but shows strong lateral and vertical wind shears.

More CAT is found over mountain ranges and over continents than over oceans (2) suggesting that the roughness of the terrain provides a source of perturbation energy which acts even up to stratospheric flight levels. Most CAT areas are of only small horizontal extent ( $<10$ miles in diameter), although a few cases are on record where CAT extended over wide regions ( $>200$ miles). The vertical extent of turbulent layers usually is shallow $(>3000$ $\mathrm{ft}$, although at times they may reach considerable thickness $(15,000 \mathrm{ft})$.

The patchiness of CAT is the cause for the difficulties which we experience in forecasting this phenomenon accurately. The average dimensions of CAT areas suggest that the origin of turbulence lies in the mesostructure of the atmosphere which defies analysis and forecasting from the macroscale tool of radiosonde observations. At best we may specify certain loose correlations between macroscale flow patterns, such as merging jet streams (5) and troughs $(3,4)$ and CAT, hoping that somehow the atmosphere provides a continuous "chain of events" between macroscale flow, mesoscale perturbations, and microscale turbulence. 


\section{METHODS OF CAT RESEARCH}

Most of our knowledge on the statistical distribution of CAT over geographical regions, with seasons, and with upper-atmospheric flow patterns stems from pilot reports. These are usually classified into 5 intensity groups between light and severe turbulence. A certain amount of subjectivity adheres to these reports. Nevertheless they proved to be invaluable for estimating turbulence expectancy on a statis tical basis.

Specially instrumented aircraft provide the most reliable information on CAT. Two essentially different approaches may be taken in such aircraft measurements:

(1) The aircraft may be used as a "sensor", by measuring its accelerations caused by atmospheric gusts. This approach requires a detailed knowledge of the aircraft's response to atmospheric turbulence over a wide range of the frequency spectrum of vibrations. This is usually done by corsidering the spectrum of aircraft accelerations--the output spectrum $\mathrm{E}_{\mathrm{O}}(\mathrm{k})$ - -as being caused by the input spectrum $E_{i}(k)$ of atmospheric gusts, modified by the frequency response function $\mathrm{T}(\mathrm{k})$ of the aircraft. The relationship may be expressed as

$$
E_{o}(k)=/ T(k) / 2 E_{i}(k)
$$

$\mathrm{k}$ is the wave number of the vibrations.

Since both vertical and horizontal gusts will result in vertical and horizontal accelerations of the aircraft, some arbitrary assumptions usually have to be made on the shape of gusts. This introduces some uncertainty into the interpretation of measurements, even though one may assume that the vertical (w) component of gusts predominates in the record of vertical accelerations. Most of the Russian (6, 7, 8) and American (9) CAT records were obtained in this way.

(2) Using the aircraft as an "inertial" platform circumvents this difficulty to a large extent. Atmospheric gusts are measured by sensitive pressure probes as in the TOPCAT measurements over Australia (10, 11, 12) or by vanes (13) mounted on a nose boom. Motions of the aircraft will simulate atmospheric gusts in the records. They may be corrected for, however, by registering accelerations and attitude data of the aircraft and by subtracting the aircraft motions thus derived from the gust measurements. No assumptions on the threedimensional shape of the gusts is necessary. Therefore, all three components ( $u, v$, and $w)$ of atmospheric gusts may be studied in a relatively wide frequency range.
Aside from the measurements described above, data on turbulence in the free atmosphere may also be obtained by other means. Large-scale velocity variations in the atmosphere may be considered as some sort of turbulence--although they will not be felt as CAT. From a philosophical point of view, we may even consider the large cyclones and anticyclones which are evident from global weather maps, as a form of "turbulent eddies". This spectrum range of "turbulence" with wave lengths of several hundred kilometers (as compared with "wave" lengths of approximately $100 \mathrm{~m}$ in (AT) may, of course, be explored conveniently by standard rawinsonde measurements.

Special flights of constant-level balloons, which are accurately tracked (14), as well as Doppler-radar wind measurements by aircraft $(15,16,8)$ provide "turbulence" data in the socalled "mesoscale" spectrum range of wave lengths between ca. $10 \mathrm{~km}$ and several hundred $\mathrm{km}$.

Backscatter (17) and forward scatter (18) of electromagnetic waves of various characteristics (from laser to long-wave radar) may provide turbulence information at very short wave lengths (from tens of meters to millimeters).

All these various sets of data may be pieced together in order to gain some insight into the laws of turbulence in the free atmosphere. As may be seen, CAT occupies only a short section of this whole spectrum of turbulent motions--similar to the visible light being only a small section of the spectrum of electromagnetic waves.

\section{SPECTRA OF TURBULENCE}

From turbulence theory it follows that the kinetic energy of turbulent motion in the wave number range $\mathrm{k}$ to $\mathrm{k}+\mathrm{dk}$ may be express ed as

$$
\mathrm{E}(\mathrm{k})=\alpha^{2 / 3} \mathrm{k}^{-5 / 3}
$$

for the "inertial subrange", i. e., for turbulent motion of a scale small enough to be unaffected by buoyant forces, yet large enough so that viscous forces are still negligible. Within this range, kinetic energy is dissipated at the rate " $\epsilon$ " (rate of dissipation of energy) merely by larger eddies breaking up into smaller ones. $\alpha$ is considered to be a universal constant. Under such conditions the kinetic energy $\mathrm{E}(\mathrm{k})$ has been found proportional to the $-5 / 3$ power of the wave number $\mathrm{k}$. This is the so-called "minus five thirds law of turbulence". 
This power law may easily be expressed as a straight line of $-5 / 3$ slope in $\log -\log$ coordinate paper with log $\mathrm{k}$ plotted along the abscissa and $\log E(\mathrm{k})$ entered along the ordinate. [The dimensions of $E(k)$ are $\frac{(\text { length })^{2}}{\left(\text { time }^{2}\right.} / \frac{\text { cycles }}{\text { length }}$ the dimensions of $\mathrm{k}$ are (length) ${ }^{-1}$ ].

If we wish to consider the kinetic energy contained within a wave band $k_{1}$ to $k_{2}$, we may simply integrate eqn. (2) between these two boundaries. Since the atmospheric turbulence spectrum extends over a very wide range of wave numbers, it is convenient to retain the abscissa in the form of $\log \mathrm{k}$ rather than $\mathrm{k}$. It is easily seen that

$$
\int_{k_{1}}^{k_{2}} k \cdot E(k) \cdot d \ln k=\int_{k_{1}}^{k_{2}} E(k) d k
$$

Thus, a diagram which contains $\log \mathrm{k}$ as abscissa and $k \cdot E(k)$ [with dimension $\frac{(\text { length })^{2}}{(\text { time })^{2}}$ of kinetic energy] as ordinate gives a true energy representation of atmospheric turbulence.
Such a diagram is shown in Fig. 1 for measurement flights Nos. 18B and 18D of Project TOPCAT. It contains both, u (longitudinal) and $w$ (vertical) components of turbulence. If turbulent energy were decaying according to eqn. (2) by a simple transfer of energy from larger to smaller eddies, one should expect the spectral lines in this diagram to follow an exponetial curve of the form

$$
\mathrm{y}=\mathrm{a} \cdot 10^{\mathrm{bx}}
$$

where $y=k \cdot E(k), a=\alpha \epsilon^{2 / 3}, b=-2 / 3$, and $\mathrm{x}=\log \mathrm{k}$. Such a curve would look rather similar to a smooth representation of the spectrum W $18 \mathrm{D}$.

Spectral curves with steeper slopes than those shown by such an exponetial curve would indicate that turbulent energy is decaying more rapidly than by a mere breaking-up of large eddies into smaller ones, due to an additional energy sink in the atmosphere. Shallower slopes, or a reversal of slope, on the other hand, would indicate a source of turbulent energy in the atmosphere.

Figs. 2, 3 and 4 show typical turbulence

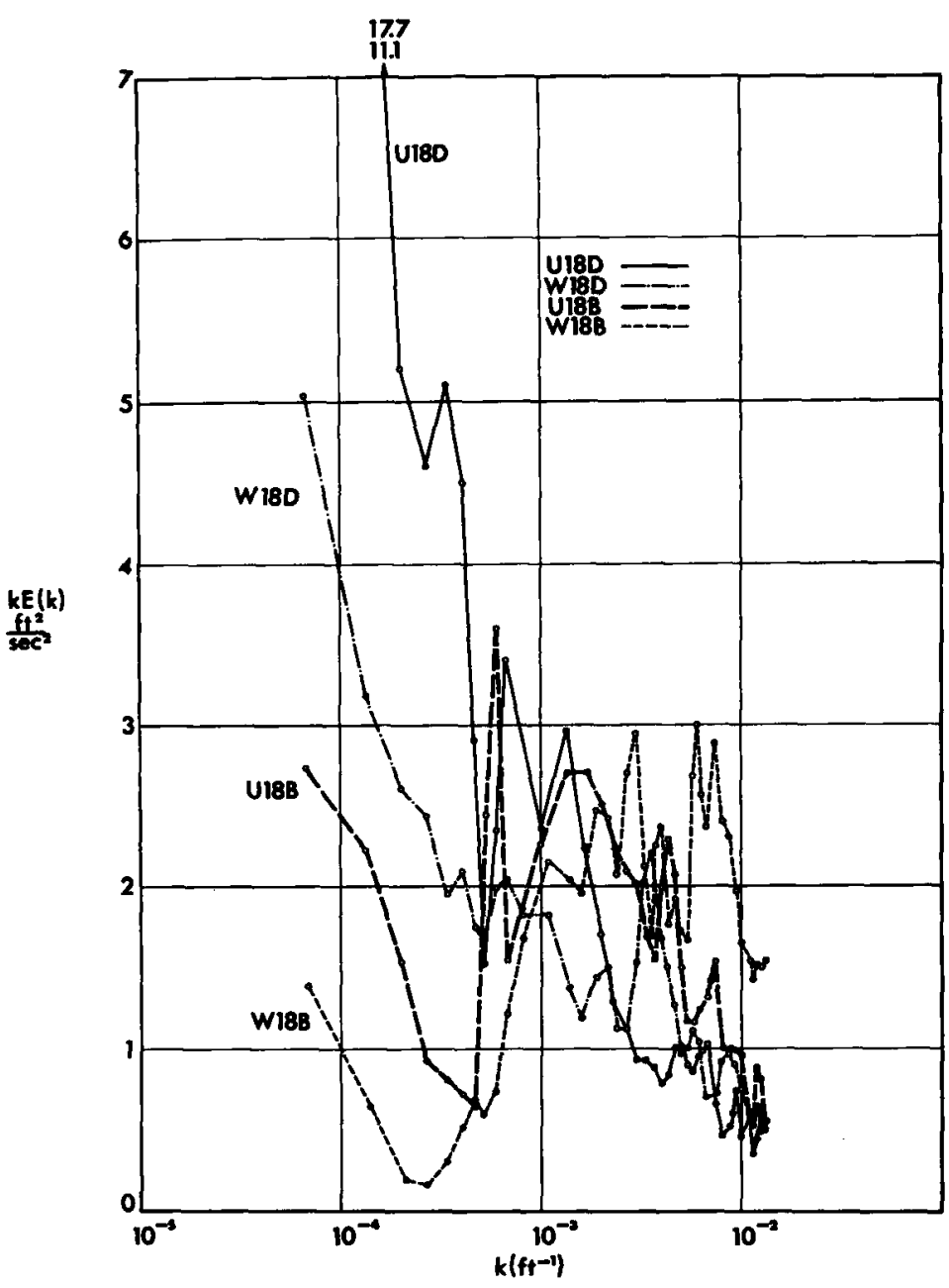

Fig. 1 - Power-spectra of $u$ - and w-component of turbulence, measured by Project TOPCAT Flight Nos. 18B and 18D over southern Australia on August 21, 1963. Ordinate contains $\mathrm{k} . \mathrm{E}(\mathrm{k})$, abscissa is log k. (12) 
spectra of the $u, v$, and $w$ components of gusts measured during Project TOPCAT in a log-log representation. Entered into these diagrams are the theoretical $-5 / 3$ slopes. The steeper -3 and $-11 / 5$ slopes pertain to theoretical speculations on impedance of turbulence under stable stratification due to buoyant forces.

\section{PHYSICAL CAUSES OF CAT IN THE ATMOS- PHERE}

Accepting the atmospheric gusts which lead to CAT as part of the turbulence spectrum of atmospheric motions, we may speculate on the difference between turbulence at high levels and near the ground or in the wind tunnel.

Most of the flight data presented above stem from stable regions in the stratosphere above, or in the troposphere underneath, the jet stream. In any case turbulence at these levels is generated by an excess of shearing stresses over thermal stability, as expressed by Richardson's turbulence criterion

$$
R i=\frac{k_{t}}{k_{m}} \frac{\frac{g}{\theta} \frac{\partial \theta}{\partial z}}{\frac{\partial V^{2}}{\partial z}}
$$

$\mathrm{k}_{\mathrm{t}}$ and $\mathrm{k}_{\mathrm{m}}$ are the exchange coefficients of heat and momentum. Their ratio usually is assumed to be one. $\theta$ stands for potential temperature, $g$ for the acceleration of gravity, $z$ for the vertical coordinate, and $V$ for wind speed. If $\mathrm{Ri}$ becomes smaller than a certain critical value (in the free atmosphere probably close to 1), turbulent flow will result.

Near the ground or near a solid boundary, turbulence is largely controlled by the roughness of this boundary surface. In the free atmosphere turbulence of small wave lengths has to be generated spontaneously without the aid of a rough surface. Only large "turbulent eddies" ( such as cyclones or anticyclones, mountain waves, etc.) are still controlled by ground effects, even at stratospheric heights. Turbulence of the wave-scale of CAT, occurring away from the friction layer near the

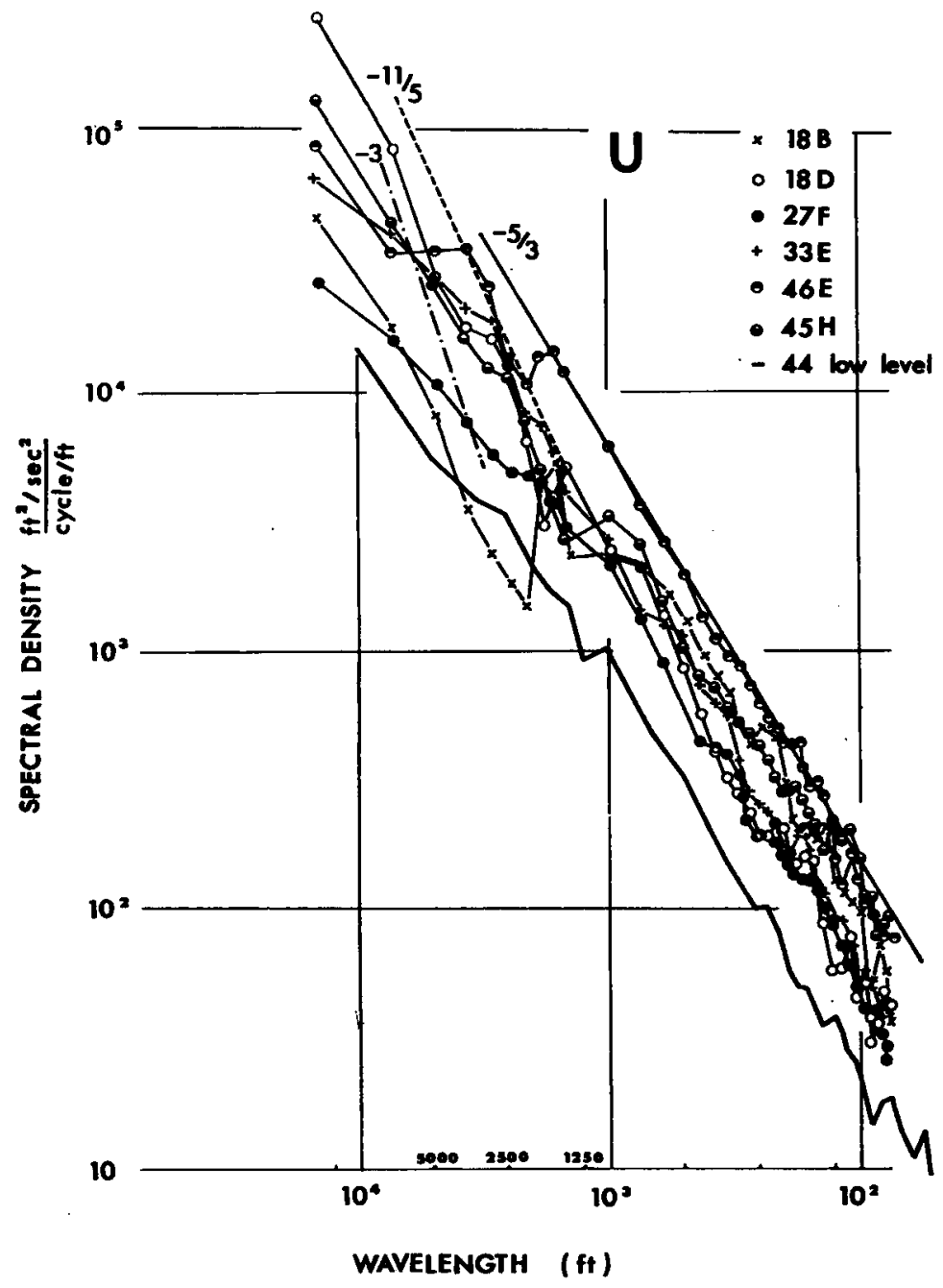

Fig 2 - Spectra of u-(longitudinal) component of turbulence measured by Project TOPCAT over Australia for flights as indicated in the diagram. Ordinate contains $\log E(k)$, abscissa is $\log k$. Slopes of $-5 / 3,-3$ and $-11 / 5$ have been entered for comparison. (12) 
ground, should be considered of spontaneous origin. The local imbalance between shear and thermal stability may, however, be controlled by meso-scale eddies which, in turn, may still be correlated with the "roughness" of the terrain. This would explain the relative prevalence of CAT over mountains and hills.

Buoyant effects in stable stratification, leading to -3 or $-11 / 5$ slopes of the spectrum curves $(19,20)$ may be of importance near the ground where turbulence is mechanically induced by roughness. In the case of CAT, however, one should not expect the existence of a "buoyant subrange" of turbulence because the very existence of turbulence in a thermally stable environment already indicates that shearing stresses have over-compensated for buoyant effects of thermal stability. In agreement with this no buoyant subrange was found over Australia from TOPCAT measurements.

Which mechanisms in the free atmosphere generate CAT? To answer this question we may inspect more closely the turbulence spectra shown in Figs. 2, 3, and 4 as well as Fig. 1. From the latter it is evident that the spectra in the CAT wave-length range

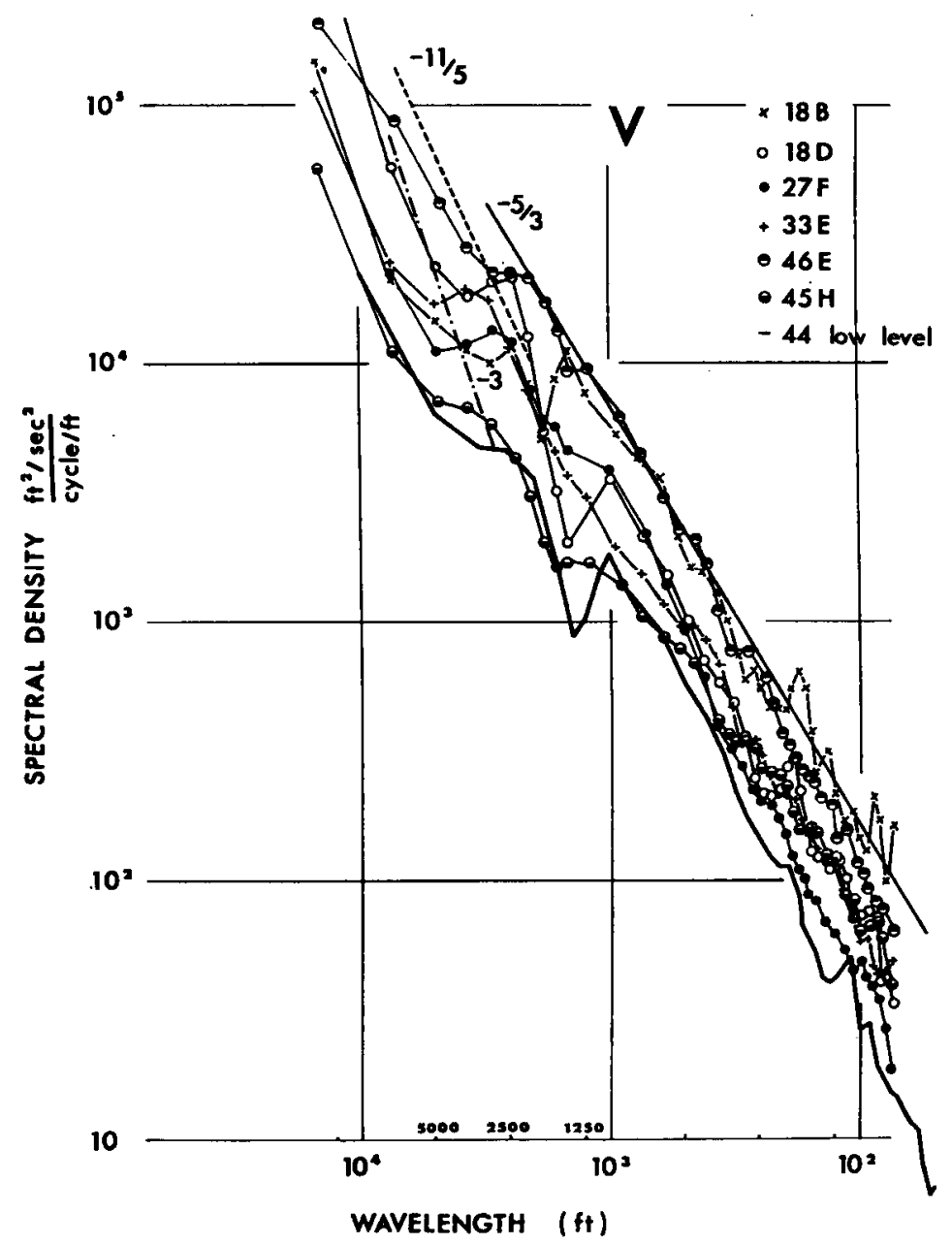

(ca. 100-600 ft) do not follow the slope of simple energy decay as given by eqn. (4). Especially the $u$ - and $w$-spectra of Flight $18 \mathrm{~B}$ show a well-developed hump, indicating that turbulent energy is added to the atmosphere between wave numbers of approximately $3 \times 10^{-4} \mathrm{ft}^{-1}$ and $5 \times 10^{-3} \mathrm{ft}^{-1}$ (i. e., between wave-lengths of ca. $3000 \mathrm{ft}$ and $200 \mathrm{ft}$ ).

This hump also appears in Fig. 4, although less well expressed due to the different coordinate system. The spectra of all flight measurements shown in Figs. 2 to 4 show remarkably little scatter at wave lengths $<1000 \mathrm{ft}$ consistent with the moderate level of turbulence experienced in all these flights. The spectra also align fairly closely to a $-5 / 3$ slope in this short-wave length range. At larger wave lengths the w-spectra show considerably more scatter than the $u$ - and $v-s p e c-$ tra. Especially the spectra of Flights 18B, $27 \mathrm{~F}$ and $45 \mathrm{H}$ appear to consist of two different segments, a short and a long wave-length one, connected by a hump in the spectral curve. This hump provides valuable clues as to the origin of CAT.

We may use Flights $18 \mathrm{~B}$ and $18 \mathrm{D}$ as an
Fig. 3 - Same as Fig. 2, except v-(transverse) component. (12) 
example. Both were flown on 21 August 1963 near 29, $000 \mathrm{ft}$ pressure altitude, and near $31^{\circ} 40^{\prime} \mathrm{S}$ and $140^{\circ} 27^{\prime} \mathrm{E}$ (i. e., the area to the east of the Flinders Range, South Australia, a range of low mountains of ca. $3000 \mathrm{ft}$ elevation). Both sets of spectra were obtained from the same turbulence region within minutes from each other. Nevertheless, the long-wave portions, especially of the w-spectra, differ appreciably in kinetic energy, as may be seen from Fig. 5, which contains these spectra in a smoothed form.

The main difference between the two measurements consists in the angle between course and wind direction. [Similar changes in spectral characteristics with changes in this angle were observed in other pairs of flights $(11,12)]$. During Flight 18B, flown almost perpendicular to the wind, the spectral density at long waves is drastically reduced as compared with $\mathrm{E}(\mathrm{k})$ measured during Flight 18D flown parallel to the wind. The conclusion to be drawn is that a wave phenomenon was encountered by the aircraft, the wave crests and troughs in the present case showing a preferred orientation nearly normal to the wind direction.
As the aircraft flies almost parallel to the wave crests (i. e., normal to the wind), it reduces the effect of up-and down-drafts at the particular wave lengths of the wave phenomenon.

(As a comparison, one might consider a surfer riding the slope of a wave). As the aircraft turns into the wind (Flight 18B), it experiences the full force of vertical motion associated with the waves (like a speed boat racing over rough water).

The "waves" thus detected by the aircraft are not "clean" or sharply defined in terms of one particular wave length. If they were, one should observe one single spike in the energy spectrum associated with this particular wave length or wave number. Instead these waves are apparently irregularly shaped and encompass a rather broad band of wave lengths. One might compare them with aircraft observations of the sometimes irregular waves appearing on the top side of a stratus overcast.

These waves apparently break down into isotropic turbulence and eddies of smaller proportions which are ultimately experienced as $\mathrm{CAT}$ by the measuring aircraft.

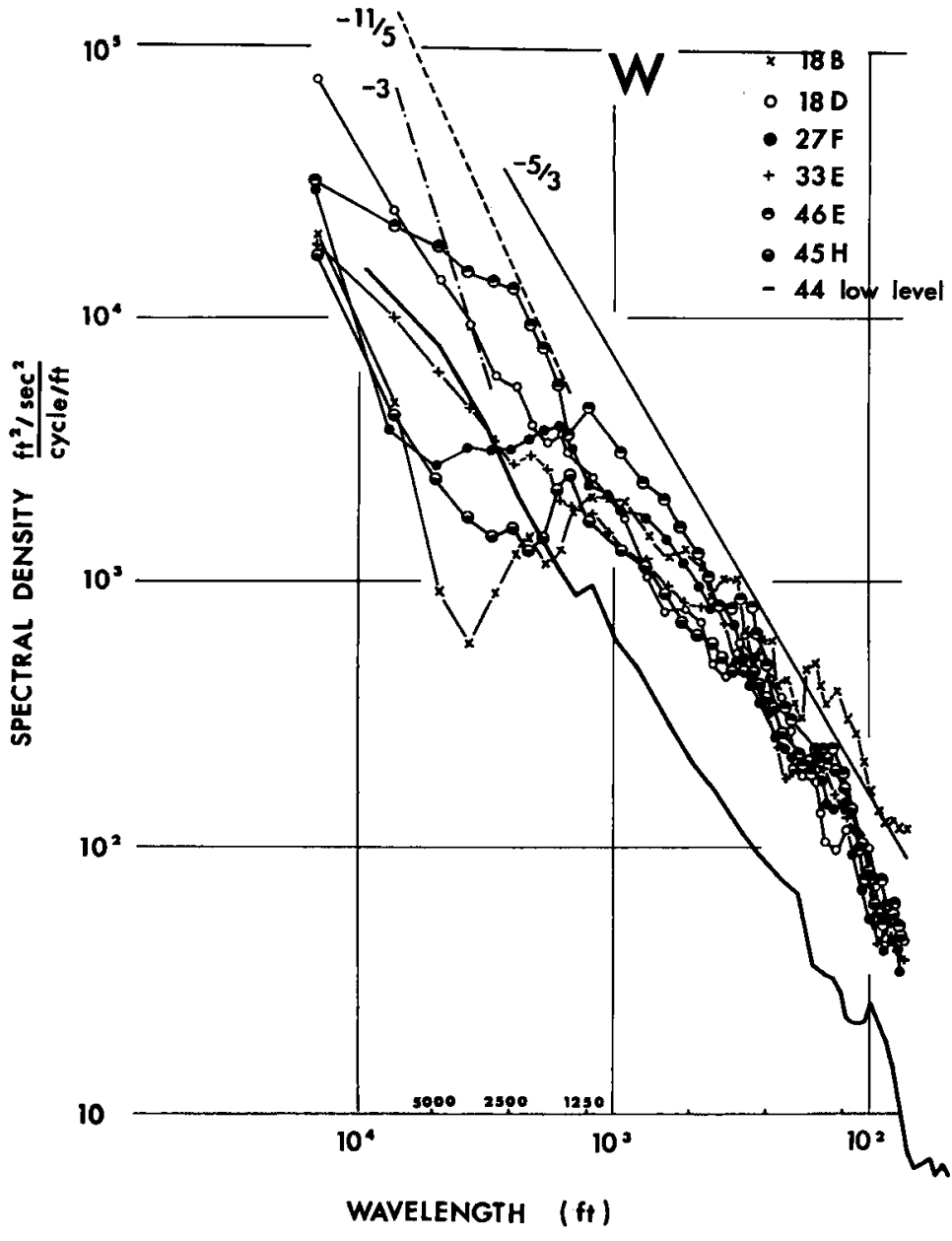

Fig. 4-Same as Fig. 2, except $\mathrm{w}$-(vertical) component. (12) 
THE FEASIBILITY OF REMOTE SENSORS IN CAT DETECTION

Since forecasting the precise location of CAT areas from standard meteorological data is obviously impossible because of the different scales involved, attempts have been made to recognize CAT patches sufficiently far ahead of the aircraft in order to allow evasive maneuvers.

Experiments with a temperature sensor conducted by Eastern Airlines (21) showed a certain correlation between temperature changes along the flight path and CAT occurrence. Such correlation should be expected in the "jet stream front" underneath the jet core when it is traversed nearly perpendicular to the flow. If an aircraft flies parallel to the jet stream, as would be expected with west-east oriented flight routes, the aircraft may stay in the vicinity of the jet-stream front for considerable time without experiencing noticeable temperature changes. Yet it may encounter CAT in this region. Temperature sensors, therefore, do not seem to offer a universally adaptable solution to the problem of CAT warning. Furthermore, the length of time between the beginning of any observed temperature change and the occurrence of CAT (if any) may not be sufficient to allow evasive maneuvers.

More sophisticated remote CAT detection may be entirely feasible if the sensors make use of the physical structure of the phe- nomenon. Since CAT in the upper troposphere and stratosphere seems to be associated mostly with stable regions and shearing discontinuities, the backscatter and/or forward scatter of electromagnetic waves from such discontinuities may be utilized. The latter would probably have to rely on ground-based instrumentations, while sensors of the former kind may be carried by the aircraft. Instrumentation of this kind theoretically may range over a wide spectrum of wave-lengths from laser to longwave radar. It should be kept in mind, however, that the discontinuities which one would look for may not be sharply defined as in the case of the top of convective bubbles or sharp inversions with large changes of refractive index over short distances along the vertical coordinate. The action of CAT may actually have diluted any sharp discontinuity that may have existed at one time, and we may find a zone of gradual transition in wind speeds and temperatures instead.

Instead of measuring the scattering properties of turbulent regions, it might be just as feasible to detect by remote sensors the original atmospheric structure that led to turbulence. Vertical discontinuities in temperature lapse rate, for instance, might be detected by infrared radiometers. The remote measurements of vertical wind shear may present a more difficult problem to solve. A combination of the two, however, would give a direct indication of Richardson's number ahead of

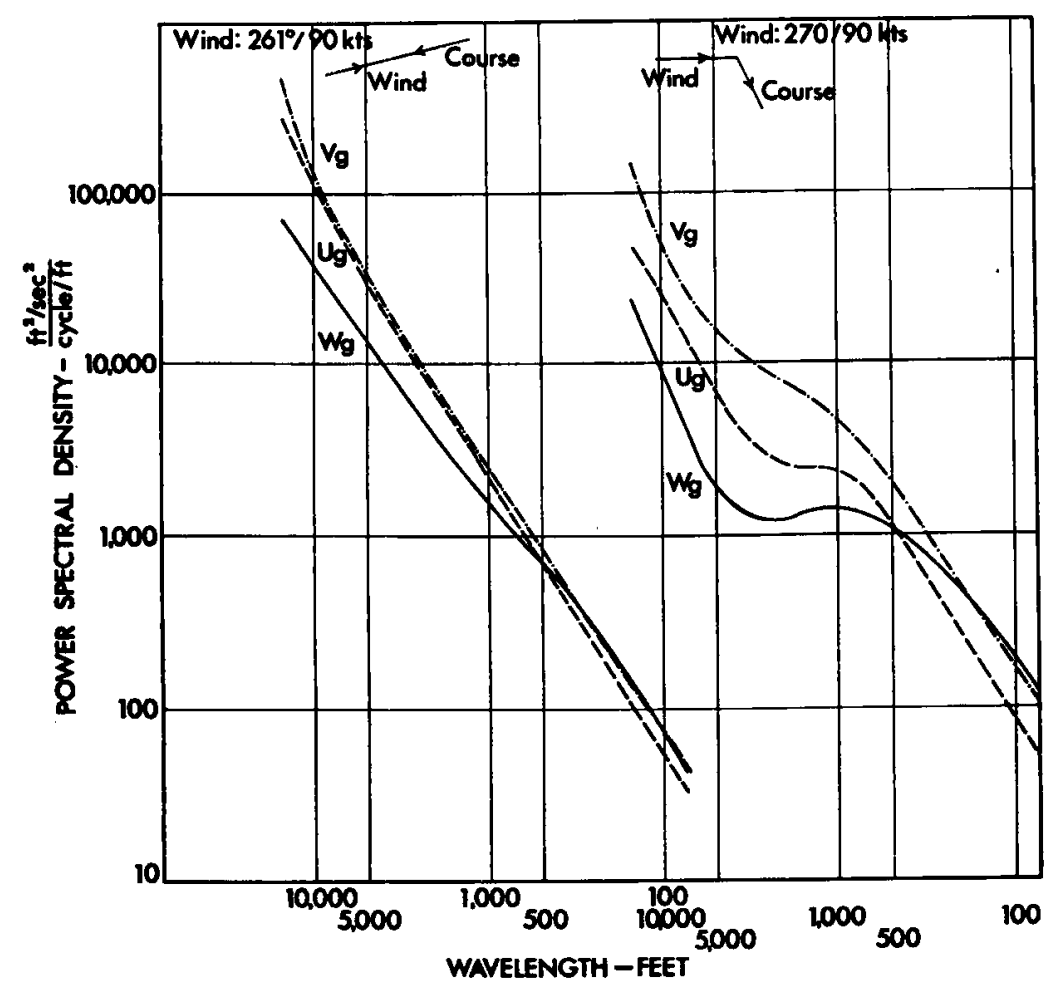

Fig. 5 - Smoothed spectra of TOPCAT Flight Nos. 18D (left-hand side) and $18 \mathrm{~B}$ (right-hand side). Mean wind direction and course are indicated on top of each diagram. (After: (10)) 
the aircraft and thus would give an indication of the atmosphere's readiness to provide turbulence energy.

\section{FUTURE PROGRAMS}

Even though we have come to recognize the physical processes leading to CAT in the stratosphere and in tropospheric stable layers as a breakdown of gravity waves into isotropic turbulence, we still have to answer many questions.

We do not yet know the precise mechanism by which such a breakdown occurs. We do not know the conditions that govern the degree of resulting CAT--light, moderate or severe. Finally, the development of remote detectors of CAT is still in its infancy stages.

From all this it appeats that additional and more sophisticated flight measurements of turbulence will be necessary together with the testing of remote sensors, such as infrared radiometers, radar, etc. Specifications for such measurements will have to contain detailed and continuous records of atmospheric gusts in three coordinate components, with a time resolution of hundredths of seconds. In addition, wind and temperature will have to be recorded on a continuous basis.

In order to explore the environment of CAT regions, two--preferably three-aircraft should be deployed. Gust-measurement capabilities would be necessary for only one airplane, however. The meso- and micro-scale of the vertical temperature and wind structure could be measured by such simultaneous measurement flights. This will yield data which would allow conclusions on the formation mechanism of gravity waves and their breakdown into CAT.

Such flights should be conducted under various conditions of upper flow patterns and terrain, to obtain a grasp on the mesoscale structure of the atmosphere which provides the energy source for CAT. The additional use of unscheduled radiosonde ascents and of constant-density balloons should be contemplated.

Efforts should be made to combine such measurement flights with tests of remote CAT sensors. Only in this way will it be possible to obtain a precise indication of the physical causes of clear-air returns registered by these sensors, and to make any correlation of such records with CAT meaningful.

It appears that such concerted efforts of attacking the problem of CAT requires careful planning of instrument packages, logistics, forecasting techniques and procedures, etc.-assuming that the necessary high priority of aircraft availability can be obtained. Furthermore, techniques and schedules of data reduction and interpretation will have to be outlined even before such an investigation is started. This should help to avoid the common complaint against such field operations that their goal is achieved as soon as the data are stored away in filing drawers "for future reference".

The logical conclusion would be that various government agencies would pool their efforts with private parties and universities interested in this particular field of atmospheric physics because one large and wellplanned operation in the long run will be far less expensive than a number of small efforts with only limited results.

\section{REFERENCES}

1. J. K. Bannon, "Weather Systems Associated with Some Occasions of Severe Turbulence at High Altitude." Meteorol. Mag. London, 81: 97-101, 1952.

2. J. Clodman, G. M. Morgan, Jr. and J. T. Ball, "High Level Turbulence." Air Weather Service, Technical Report 158, 1961.

3. DeVer Colson, "Analysis of ClearAir Turbulence During April 1960. " Monthly Weather Review, 89: 94-98, 1961.

4. DeVer Colson, "Analysis of ClearAir Turbulence Data for March 1962. " Monthly Weather Review, 91 (2): 73-82, 1963.

5. E. R. Reiter and A. Nania, "JetStream Structure and Clear-Air Turbulence (CAT)." J. of Applied Meteorol., 3(3): 247$260,1964$.

6. G. N. Shur, "Experimental Investigation of the Energy Spectrum of Atmospheric Turbulence." Trudy TsAO, No. 43, 1962.

7. N. K. Vinnichenko, N. Z. Pinus, and G. N. Shur, "Some Results of the Experimental Turbulence Investigations in the Troposphere." Paper presented at the International Colloquium on the Fine-Scale Structure of the Atmosphere, Moscow, June 15-22, $196 b$.

8. N. Z. Pinus, E. R. Reiter, G. N. Shur, and N. K. Vinnichenko, "Power Spectra of Turbulence in the Free Atmosphere." Manuscript submitted to "Tellus", 1965.

9. R. H. Rhyne and R. Steiner,

"Turbulence and Precipitation Problems Associated with Operation of Supersonic Transports. " Paper presented at the Fourth Conference on Applied Meteorology, Hampton, Virginia, September 10-14, 1962.

10. A. Burns and C. K. Rider, "Project TOPCAT, Power Spectral Measurements 
of Clear-Air Turbulence Associated with Jet Streams." Royal Aircraft Establishment, Technical Memo. No. Structures, 1965.

11. E. R. Reiter and A. Burns, "Atmospheric Structure and Clear-Air Turbulence. Colorado State University, Atmospheric Science Technical Paper No. 65, 1965.

12. E. R. Reiter and A. Burns, "The Structure of Clear-Air Turbulence Derived from TOPCAT Aircraft Measurements. " Manuscript submitted to J. of Atmosph. Sci., 1965 .

13. W. Crooks, "High Altitude ClearAir Turbulence." Interim Report, Flight Dynamics Laboratory, Research and Technology Division, Air Force Systems Command, WrightPatterson Air Force Base, Ohio, 1965.

14. H. T. Mantis, "The Structure of Winds of the Upper Troposphere at Mesoscale. " J. of Atmosph. Sci., 20 (2): 94-106, 1963.

15. S. - K. Kao and H. D. Woods, "Energy Spectra of Meso-Scale Turbulence along and across the Jet Stream. "J. Atmosph. Sci., 21 (5): 513-519, 1964.

16. N. Z. Pinus, "Statistical Characteristics of the Horizontal Component of the Wind Velocity at Heights of $6-12 \mathrm{~km}$. "Bulletin of the Academy of Sciences, USSR, Geophys. Ser., 105-107, 1963.

17. D. Atlas, K. R. Hardy, and K. M. Glover, "Multi-wavelength Backscatter from the Clear Atmosphere. " Paper presented at the International Colloquium on the Fine-Scale Structure of the Atmosphere, Moscow, June 15-22, 1965 .

18. I. Revah and A. Spizzichino,

"Etudes des stratifications horizontales dans la haute atmosphere. " Paper presented at the International Colloquium on the Fine-Scale Structure of the Atmosphere, Moscow, June 15-22, 1965 .

19. R. Bolgiano, Jr., "Turbulent Spectra in a Stably Stratified Atmosphere. " J. Geophys. Res., 64: 2226, 1959.

20. R. Bolgiano, Jr., "Structure of Turbulence in Stratified Media. " J. Geophys. Res., 67: 3015-3123, 1962.

21. P. W. Kadlec, "A Study of Flight Conditions Associated with Jet Stream Cirrus, Atmospheric Temperature Change and Wind Shear Turbulence. " Final Report to U. S. Weather Bureau under Contract No. Cwb $10674,1964$. 